Ciência Florestal, Santa Maria, v. 5, n. 1, p. 33-54, 1995

ISSN 0103-9954

\title{
ANÁLISE FITOSSOCIOLÓGICA DE ALGUMAS ÁREAS REMANESCENTES DA FLORESTA DO ALTO URUGUAI, ENTRE OS RIOS IJUÍ E TURVO, NO RIO GRANDE DO SUL
}

\author{
PHYTOSOCIOLOGICAL ANALYSIS OF SOME REMAINING \\ NATURAL STANDS IN ALTO URUGUAI FOREST, BETWEEN RIVERS \\ IJUÍ AND TURVO, RIO GRANDE DO SUL STATE - BRAZIL
}

\author{
Sandro Vaccaro ${ }^{1}$ Solon Jonas Longhi ${ }^{2}$
}

\begin{abstract}
RESUMO
Para descrever a composição florística e estrutura de algumas áreas remanescentes da floresta do Alto Uruguai foram levantadas oito unidades amostrais de 15 x 100 m entre os rios Ijuí e Turvo, nos municípios de Roque Gonzales, Porto Lucena, Porto Xavier, Alegrim, Tuparendi, Horizontina, Criciumal e Três Passos, no Estado do Rio Grande do Sul. Nas parcelas foram consideradas todas as árvores vivas com circunferência a altura do peito maior ou igual a $30 \mathrm{~cm}$, onde mediram-se a circunferência, altura do fuste e altura total. Foram amostrados 588 indivíduos distribuídos em 26 famílias, 53 gêneros e 66 espécies. As famílias mais importantes foram Leguminosae, Boragineceae e Sapindaceae e as espécies mais importantes foram Patagonula americana, Diatenopteryx sorbifolia, Helietta longifoliata, Luehea divaricata, Holocalyx balansae e Chrysophyllum gonocarpum. Os fragmentos florestais analisados demonstram estar se regenerando de perturbações sofridas no passado.
\end{abstract}

Palavras-chave: fitossociologia; Floresta Estacional Decidual; Alto Uruguai.

1 Engenheiro Florestal, Mestrando do Programa de Pós-graduação em Engenharia Florestal, Centro de Ciências Rurais, Universidade Federal de Santa Maria, Av. Roraima, 1000, CEP 97105-900, Santa Maria (RS), Brasil.

2 Engenheiro Florestal, MSc., Professor Titular do Departamento de Ciências Florestais, Centro de Ciências Rurais, Universidade Federal de Santa Maria, Av. Roraima, 1000, CEP 97105-900, Santa Maria (RS), Brasil. 


\begin{abstract}
In order to describe the floristic composition and the present structure of the natural vegetation eight $15 \times 100 \mathrm{~m}$ plots were laid down in Alto Uruguai forest, between rivers Ijuí and Turvo, in the countryside of Roque Gonzales, Porto Lucena, Porto Xavier, Alecrim, Tuparendi, Horizontina, Crisciumal and Três Passos, in Rio Grande do Sul state, southern Brazil. In the plots, all the alive trees with the circumference at the chest height or equal to $30 \mathrm{~cm}$ high were considered, where it was measured the circumference, at the height of the shaft and the total height. It was sampled 588 individuals distributed in 26 families, 53 genera and 66 species. The most important families were Leguminosae, Boragineceae and Sapindaceae and the most important species were Patagonula americana, Diatenopteryx sorbifolia, Helietta longifoliata, Luehea divaricata, Holocalyx balansae and Chrysophyllum gonocarpum. The phytosociological structure was found to be severely modified with respect to the original or ancient structure.
\end{abstract}

Keywords: phytosociology; Deciduous Seasonal Forest; 'Alto Uruguai'.

\title{
INTRODUÇÃO
}

A floresta do Alto Uruguai, considerada por RAMBO (1956a) como um prolongamento empobrecido da mata subtropical da bacia do Rio Paraná, foi designada tecnicamente por IRGANG (1980) de Mata Pluvial Subtropical Perenifolia do Alto Uruguai. KLEIN (1984), tomando como base o aspecto fisionômico das florestas do sul do pais, concluiu que esta formação pertence a uma Floresta Estacional Decidual; termo também empregado no projeto RADAMBRAISL (PASTORE \& RANGEL FILHHO, 1986). e no recente Mapa de Vegetação do Brasil (IBGE, 1993).

De acordo com RAMBO (1956B), no Rio Grande do Sul a mata do Alto Uruguai começava nas margens do rio Jacuí; desenvolvia-se no extremo noroeste no maior núcleo de mato fechado do Estado; ligava-se, entre Passo Fundo e Lagoa Vermelha, ao longo dos afluentes do rio Taquari, a mata da Fralda da serra geral; e reduzia-se a um cordão marginal no rio Pelotas. KLEIN (1972) complementa que sua ramificação prolongava-se por todos os vales e afluentes do Rio Uruguai, até altitudes compreendidas entre 500 e 600 $\mathrm{m}$, onde entrava em contato com a mata dos pinhais.

Conforme KLEIN (1972), a floresta do Alto Uruguai caracteriza-se

Ci. Fl., v. 5, n. 1, 1995 
principalmente por apresentar elevada percentagem de espécies exclusivas da floresta subtropical, número relativamente pequeno de espécies arbóreas altas e sobretudo pela quase ausência de epífitas. Quanto a estrutura, afirmar que podem ser distinguidos três estratos arbóreos, além de um estrato arbustivo e um herbáceo. O estrato das árvores altas (30 a $40 \mathrm{~m}$ de altura), que que constituem a cobertura superior, não formam uma cobertura contínua, ocorrendo, muitas vezes, como árvores emergentes. A maioria das espécies componentes são deciduais ou semideciduais, e grande parte das madeiras de qualidade pertencem a este estrato. $\mathrm{O}$ estrato das árvores, comumente entre 15 a $25 \mathrm{~m}$ de altura, é bastante denso e continuo, sendo constituído, em sua maioria, por espécies perenifólias. O estrato das arvoretas é formado por um numero relativamente pequeno de árvores medianas, normalmente com 6 a $15 \mathrm{~m}$ de altura.

Atualmente, esta formação florestal no Rio Grande do Sul encontra-se fragmentada em pequenas manchas esparsas entre lavouras, principalmente as de milho, trigo e sobretudo soja, além da área de 17.491 ha preservada do Parque Estadual do Turvo. O Inventário Florestal Nacional - Rio Grande do Sul (BRASIL, 1983) estimou existirem apenas 69.603 ha de Floresta Estacional Decidual no Alto Uruguai, valor insignificante considerando que a região fisiográfica do Alto Uruguai, com 2.606.200 ha de área, segundo FORTES (1956), era coberta na sua maior parte por este tipo florestal. LEITE \& KLEIN (1990) comentam que os poucos povoamentos residuais de Florestas estacionais na Região Sul do Brasil (cerca de 4,2\% do total original) encontram-se alterados e parcialmente descaracterizados. MARCHIORI (1991) cita que além da inexpressiva área ocupada pelas matas nativas privadas neste Estado, deve ser destacado o verdadeiro saque nelas perpetrado ao longo do tempo, constituindo estas, hoje, apenas pálidas sombras do que foram no passado.

Estudos quali-quantitativos da floresta do Alto Uruguai ainda são escassos. Os trabalhos realizados por ALBUQUERQUE (1977, 1985), BRACK et al. (1985), DIAS et al. (1992), IRGANG (1980), KLEIN (1972), RAMBO (1956a) e SECRETARIA DA AGRICULTURA (1980) trataram de descrições fisionômicas e da composição florística ou salientaram a importância da preservação dos remanescentes. O aspecto quantitativo foi apresentado por BRASIL (1983), no Inventário Florestal Nacional - Rio Grande do Sul, e por VASCONCELLOS et al. (1992), que desenvolveram um estudo fitossociológico no Parque Estadual do Turvo. O objetivo deste 
Vaccaro, S.; Longhi, S.J.

trabalho é analisar a composição florística e a estrutura fitossociológica de remanescentes da floresta do Alto Uruguai, ocorrentes entre os rios Ijuí e Turvo, no Rio Grande do Sul.

\section{MATERIAL E MÉTODOS}

A área de estudo localiza-se na antiga micro-região colonial de Santa Rosa (RS), entre os rios Ijuí e Turvo, afluentes do rio Uruguai (Figura 1).

A região apresenta um clima do tipo Cfa de Koppen - clima temperado, chuvas bem distribuídas ao longo do ano e temperatura média do mês mais quente superior a $22^{\circ} \mathrm{C}$ (Figura 2).

O solo da região é derivado de rochas efusivas básicas da Formação Serra Geral, apresentando basalto como substrato. Os solos predominantes pertencem as associações Ciríaco - Charrua e Pedregal-Escobar-Afloramento de Rocha e as Unidades de Mapeamento Charrua, classificados como Litólico Eutrófico; e Santo Angelo, classificado como Latossolo Roxo Distrófico. O relevo apresenta-se desde suavemente ondulado, em altitudes que variam de 80 a 400 m na Unidade de Mapeamento Santo Angelo e Associação Pedregal-Escobar-Afloramento de Rocha até relevo fortemente ondulado a montanhoso, constituído por um conjunto de formações que chegam a atingir $700 \mathrm{~m}$ de altitude, dando origem a vales fortemente dissecados por rios na Unidade de Mapeamento Charrua e Associação Ciríco-Charrua (BRASIL, 1973).

Para obtenção de dados fitossociologicos o método empregado foi o de parcelas com dimensões de 15 x $100,(1500 \mathrm{~m})$. Instalaram-se oito unidades amostrais em redutos florestais localizados em diferentes municípios; Roque Gonzales, Porto Lucena, Porto Xavier, Alecrim, Tuparendi, Horizontina, Criciumal e Três Passos, totalizando 1,2 ha de área amostrada. 


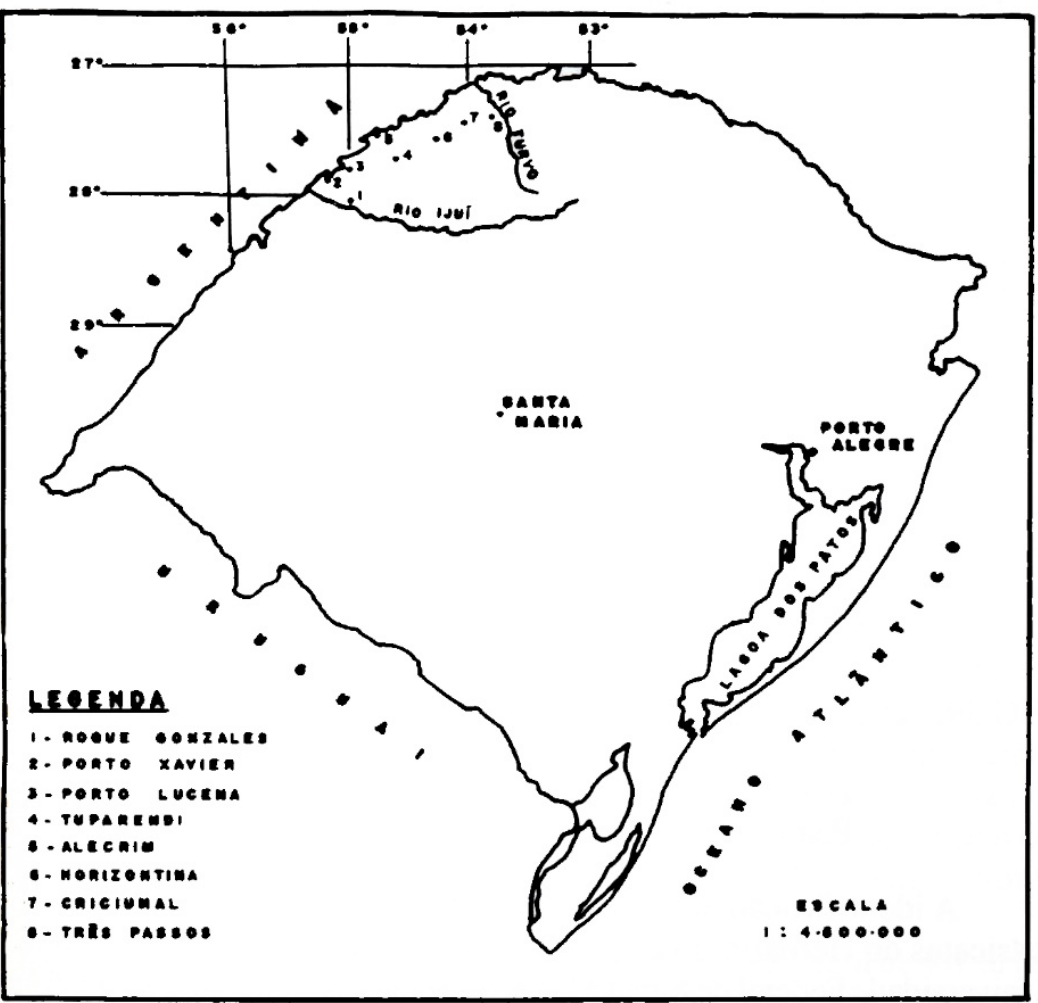

FIGURA 1: Localização da área de estudo.

FIGURE 1: Location of the study area.

Nas parcelas foram amostradas todas as árvores vivas com circunferência a altura do peito (CAP) maior ou igual a $30 \mathrm{~cm}$ (DAP maior ou igual a 9,5 cm). Em cada indivíduo mediu-se o CAP com fita métrica e estimou-se a altura do fuste (até a inserção dos primeiros galhos) e total (até o ápice) com hipsômetro de Blume-Leiss. 


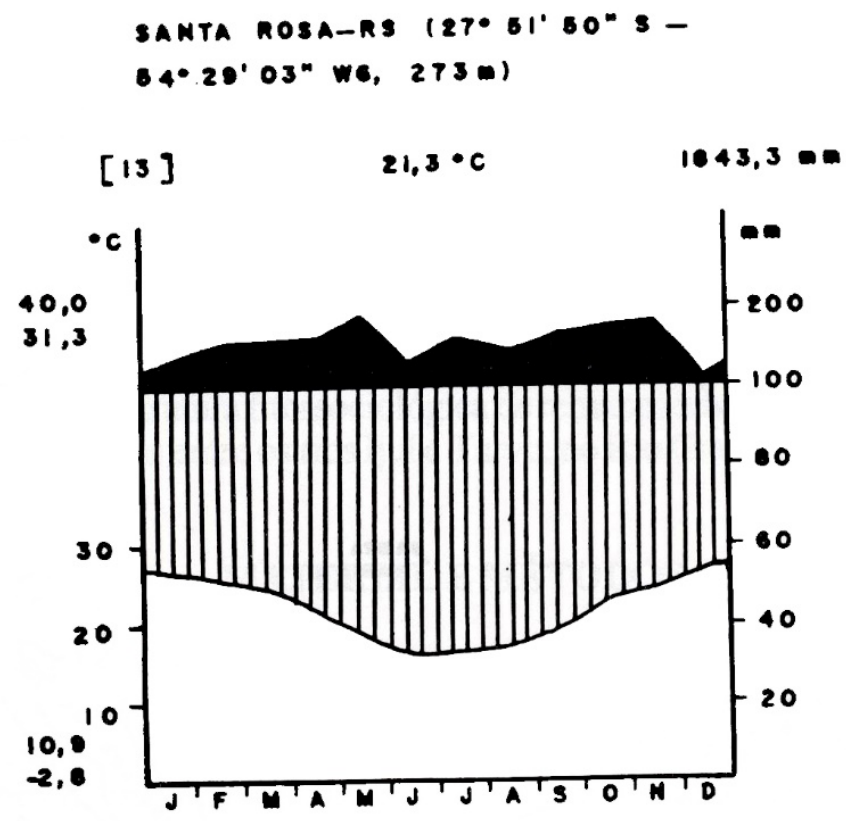

FIGURA 2: Diagrama climático da região de Santa Rosa, segundo WALTER (1963). Dados climáticos referentes ao período de 1975 a 1987. Fonte: Instituto de Pesquisas Agronômicas - DIPAGRO/ CIENTEC - Porto Alegre (RS).

FIGURE 2: Climatic diagram of Santa Rosa region, according to WALTER (1963). Climatic data referring to the period from 1975 to 1987. Source: Instituto de Pesquisas Agronômicas (Institute of Agronomic Researches) - DIPAGRO / CIENTEC - Porto Alegre (RS state).

A identificação das espécies foi feita in loco ou por comparação com exsicatas do Herbário do Departamento de Ciências Florestais (HDCF) da Universidade Federal de Santa Maria, onde também encontra-se depositado o material botânico coletado.

Os parâmetros fitossociológicos estimados foram os de densidade, frequência e dominância absolutos e relativos e índice de valor de importância (MUELLER-DOMBOIS \& ELLENBERG, 1974; MARTINS, 1979).

A estratificação foi estabelecida a partir de um histograma de 
frequência de classes de altura média com intervalo de $0,5 \mathrm{~m}$, que era a precisão a que se poderia chegar nas medições das alturas. Para a obtenção da altura média determinou-se um valor médio entre a altura do fuste e a altura total, de cada individuo amostrado.

Para a análise da distribuição diamétrica elaborou-se um histograma de frequência considerando-se um intervalo de $5 \mathrm{~cm}$ na distribuição das classes de diâmetro, exceto a terceira classe (primeira barra do histograma), cuja a amplitude foi de 9,5 a $15 \mathrm{~cm}$.

\section{RESULTADOS}

Nos $12,000 \mathrm{~m}^{2}$ representados pelas oito parcelas de $15 \times 100 \mathrm{~m}$, foram amostrados 588 individuos arbóreos com CAP mínimo de $30 \mathrm{~cm}$, correspondendo a 66 espécies identificadas distribuídas entre 53 gêneros e 26 familias. Das 66 espécies, cinco (7,6\%) foram identificadas somente até o gênero: Lonchocarpus, Ocotea, Rapanea, Rollinia e Zanthoxylon. Dos 588 individuos levantados, 21 (3,6\%) não foram identificados nem a nível de família.

A Tabela 1 lista em ordem alfabética as famílias e respectivas espécies amostradas no componente arbóreo. Leguminosae apresentou a maior riqueza florística (11 espécies), seguida das famílias Myrtaceae (7 espécies) e Lauraceae, Meliaeae e Rutaceae (5 espécies cada). Estas cinco famílias contribuíram com $50 \%$ do total de espécies encontradas; porém, 13 (50\%) famílias apresentam apenas uma espécie.

Na Figura 3, observa-se que 76,4\% dos indivíduos distribuíramse entre 8 familias. A família Leguminosae apresentou o maior número de indivíduos (79 ou 13,4\%), seguindo-se Boraginaceae com 72 (12,2\%), Sapindaceae com $67(11,4 \%)$ e Rutaceae com $62(10,6 \%)$. Quase a metade $(47,6 \%)$ das árvores amostradas pertencem a restas quatro famílias.

A densidade total absoluta e a área basal por hectare foram de 490 árvores/ha e $32,35 \mathrm{~m}^{2} /$ ha, respectivamente. $\mathrm{O}$ índice de diversidade de Shannon \& Weaver foi de 3,71, indicando que a floresta estudada apresenta alta diversidade, já que em florestas estacionais estes índices estão em torno de 3,5 .

Na Tabela 2 figuram os parâmetros de abundancia das espécies amostradas em ordem decrescente do Indice de Valor de importância (IVI). As espécies Patagonula americana, Helietta longifoliata, Diatenopteryx 
sorbifolia e Chrysophyllum gonocarpum distinguiram-se por serem as mais densas da mata, totalizando $28,09 \%$ de densidade relativa.

Das 66 espécies identificadas, 9 (13,6\%) compareceram com apenas um indivíduo. Patagonula americana, Diatenopteryx sorbifolia, Chrysophyllum gonocarpum, Helietta longifoliata e Lonchocarpus sp. foram as populações mais frequentes, sendo as duas primeiras amostradas em todas as parcelas $(\mathrm{FA}=100 \%)$, evidenciando a ampla distribuição na região em apreço. Das 66 espécies, 17 (25,8\%) ocorreram em apenas uma das unidades amostrais. Com relação a dominância destacam-se Patagonula americana, Diatenopteryx sorbifolia, Luehea divaricata, Holocalyx balansae, Ruprechtia laxiflora e Helietta longifoliata, representando juntas 53,5\% da área basal da floresta.

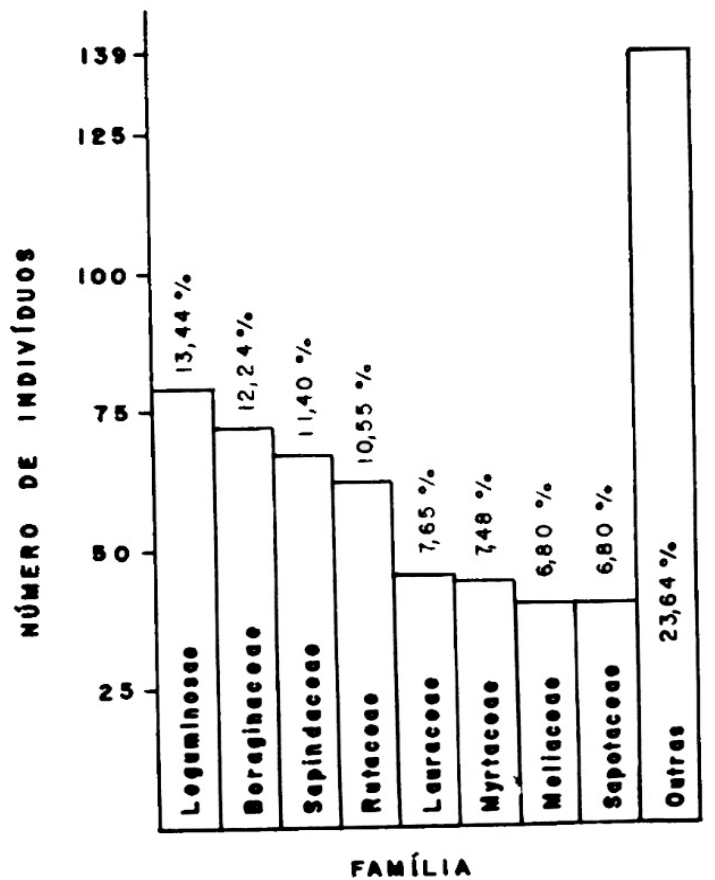

FIGURA 3: Familias com maior número de indivíduos expressos em percentagem de número total de indivíduos amostrados.

FIGURE 3: Families with the highest number of individuals expressed as percentage of the total number of sampled individuals. 
TABELA 1: Familias e espécies arbóreas amostradas em fragmentos de floresta estacional decidual do Alto Uruguai, entre os rios Ijuí e Turvo - RS. Amostras de 1,2 ha.

TABLE 1: Families and tree species sampled in fragments of deciduous seasonal rainforest of 'Alto Uruguai', between rivers Ijuí and Turvo - RS state. Samples of 1.2 ha.

\begin{tabular}{|c|c|c|}
\hline Família & Nome científico & Nome vulgar \\
\hline Anacardiaceae & Astronium balansae Engler & Pau-ferro \\
\hline Annonaceae & Rollinia sp. & Araticum \\
\hline Araliaceae & Didymopanax morototoni (Aubl.) Dcne, et Planch & Caixeta \\
\hline \multirow{3}{*}{ Bignoniaceae } & Tabeluia alba (cham) Standl. & Ipê-ouro \\
\hline & Tabeluia impetiginosa (Mart.) Standl. & Ipê-roxo \\
\hline & Tabeluia pulcherrima Sandw & Ipê-amarelo \\
\hline \multirow{3}{*}{ Boraginaceae } & Cardia ecalyculata Vell. & Louro-mole \\
\hline & Cordia trichotoma (Vell.) Arrab. ex Steud & Louro \\
\hline & Patagonia americana $\mathrm{L}$. & Guajuvira \\
\hline \multirow[b]{2}{*}{ Euphorbiaceae } & Sebastiania brasiliensis Spreng & Branquilho-leite \\
\hline & $\begin{array}{l}\text { Sebastiania commersoniana (Baill.)L.B.Smith \& } \\
\text { R.J.D }\end{array}$ & Branquilho \\
\hline \multirow{2}{*}{ Flacourtiaceae } & Banara tomentosa Clos & Guaçatunga-preta \\
\hline & Casearia sylvestris $\mathrm{Sw}$. & Chá-de-bugre \\
\hline \multirow{5}{*}{ Lauraceae } & Nectamdra lanceolata Nees et Mart. Ex Nees & Canela-amarela \\
\hline & Nectamdra megapotamica (Spreng.) Mez & Canela-preta \\
\hline & Ocotea puberula Nees & Canela-guaicá \\
\hline & Ocotea pulchella Nees et Mart. Ex Ness & Canela-lageana \\
\hline & Ocotea sp. & Canela \\
\hline \multirow{3}{*}{ Leguminosae-C. } & Apuleia leiocarpa (Vog.) Macbr. & Grápia \\
\hline & Gleditschia amorphoides (Griseb) Taubert & Açucará-faveiro \\
\hline & Holocalyx balansae Mich. & Alecrim \\
\hline \multirow{3}{*}{ Leguminosae-M. } & Albizia austrobrasilica Burk. & Angico--branco \\
\hline & Inga uruguensis Hook. \% Am. & Ingá-banana \\
\hline & Parapiptadenia rígida (Benth) Brenan & Angico-vermelho \\
\hline
\end{tabular}

Continua... 
TABELA 1: Continuação...

TABLE 1: Continued...

\begin{tabular}{|c|c|c|}
\hline Família & Nome científico & Nome vulgar \\
\hline \multirow{5}{*}{ Leguminosae-P. } & Dalbergia variabilis Vog & Rabo-de-brugri \\
\hline & Erythryna falcata Benth & Corticeira \\
\hline & Lonchocarpus sp. & Farinha-seca \\
\hline & Machaerium stipitatum Vogel & Canela-do-brejo \\
\hline & Myrocarpus fronsosus Fr. All. & Cabriúva \\
\hline \multirow{3}{*}{ Meliaceae } & Cabralea canjerana (vell.) Mart. & Canjerana \\
\hline & Cedrela fissillis Vell & Cedro \\
\hline & Trichilia catiguá A. juss & Catiguá \\
\hline \multirow{2}{*}{ Moraceae } & Trichilia clausseni C. DC. & Catiguá-vermelho \\
\hline & Trichilia elefans A. Juss. & Catiguá-ervilha \\
\hline \multirow{2}{*}{ Myrsinaceae } & Ficus luschnathiana (Miq.) Miq. & Figueira-do-mato \\
\hline & Sorocea bonplandii (Baill.) Burger, Laij. \& Boer & Cincho \\
\hline \multirow{7}{*}{ Myrtaceae } & Rapanea umbellata (Mart. ex A. DC.) Mez & Capororocão \\
\hline & Rapanea sp & Capororoca \\
\hline & Blepharocalyx salicifolius(H. B. K.) Berg & Murta \\
\hline & Britoa guazumaefolia (Camb.) Legr. & Sete-capotes \\
\hline & Campomanesia xanthocarpa Berg & Guabiroba \\
\hline & Eufenia involucrata DC. & Cereja-do-mato \\
\hline & Eugenia uniflora $L$. & Pitangueira \\
\hline Palmae & Myrcianthes pungens (Berg) Legr. & Guabijú \\
\hline Phytolaccaceae & Myrciaria rivularia $\mathrm{Camb}$ & Guapuriti \\
\hline Polygonaceae & Syagrus romanzoffiana (Cham.) Glass. & Gerivá \\
\hline Rhamnaceae & Phytolacca dioica $\mathrm{L}$. & Umbú \\
\hline Família & Nome científico & Nome vulgar \\
\hline Rubiaceae & Ruprechtia laxiflora Meiss. & Marmeleiro \\
\hline
\end{tabular}

Continua...

Ci. Fl., v. 5, n. 1, 1995 
TABELA 1: Continuação...

TABLE 1: Continued...

\begin{tabular}{|c|c|c|}
\hline Família & Nome científico & Nome vulgar \\
\hline \multirow{5}{*}{ Rutaceae } & Scutia buxifolia Reiss & Coronilha \\
\hline & Guettarda uruguensis Cham. et Schlecht. & Veludinho \\
\hline & Balfourodendron riedelianum (Engl.) Emgl. & Pau-marfim \\
\hline & Helietta longifoliata Britton & Canela-de-veado \\
\hline & Pilocarpus pennatifolius Lemaire & Cutia \\
\hline \multirow{4}{*}{ Sapindaceae } & Zanthoxyllum rhoifolium Lam. & $\begin{array}{l}\text { Mamica-de- } \\
\text { cadela }\end{array}$ \\
\hline & Zanthoxyllum sp. & $\begin{array}{l}\text { Mamica-de- } \\
\text { cadela }\end{array}$ \\
\hline & Allophylus edulis (St. Hil) Radlk. & Chal-chal \\
\hline & Cupania vernalis $\mathrm{Camb}$ & $\begin{array}{l}\text { Camboata- } \\
\text { vermelho }\end{array}$ \\
\hline \multirow{4}{*}{ Sapotaceae } & Diatenopteryx sorbifolia Radlk & Maria-preta \\
\hline & Matayba elaeagnoides Radlk. & Camboatá-branco \\
\hline & Chrysophyllum gonocarpum (Mart. \&Eich ) Emgl. & Aguaí-da-serra \\
\hline & Chrysophyllum marginatum (Hook. et Arn.) Radlk. & Aguaí-vermelho \\
\hline Simaroubaceae & Aeschrion crenata Vell. & Pau-amargo \\
\hline Solabaceae & Solanum sanctae-catharinae Dunal & Joá-manso \\
\hline Styracaceae & Styrax leprosus Hook. et Arn. & carne-de-vaca \\
\hline Tiliaceae & Luehea divaricata Mart. & Açoita-cavalo \\
\hline Verbenaceae & Vitex megapotamica (Spreng.) Mold & Tarumã \\
\hline
\end{tabular}

A Tabela 2 mostra que apenas seis espécies: Patagonula americana, Diatenopteryx sorbifolia, Helietta longifoliata, Luehea divaricata, Holocalyx balansae e Chrysophyllum gonocarpum, contribuíram com 34,9\% do IVI total. importância.

A Figura 4 apresenta a relação das famílias com maiores valores de

A Figura 5 mostra o histograma com a distribuição das classes de altura média das árvores amostradas. Embira este gráfico não permita uma delimitação precisa dos estratos arbóreos, é possível, através dele, admitir 
a existência de dois extratos. Assim, como extrato inferior considerou-se as arvores com altura total de $10 \mathrm{~m}$ e como extrato superior as árvores maiores que este limite. Apena um individuo ocorreu como emergente, pertencente a Cordia trichotoma, com 20,5 m de altura média da copa, $23 \mathrm{~m}$ de altura total e $33 \mathrm{~cm}$ de diâmetro.

TABELA 2: Parâmetros fitossocilogicos das espécies amostradas em 1,2 ha de floresta estacional decidual do Alto Uruguai, entre os rios Ijui e Turvio - RS, ordenadas segundo o IVI.

TABLE 2: Phytossociological parameters of the species sampled in 1.2 ha of deciduous seasonal rainforest of 'Alto Uruguai', between Ijui and Turvo rivers - RS state, ordered according to IVI.

\begin{tabular}{|c|c|c|c|c|c|c|c|c|c|}
\hline Pos & Espécie & & ind & DA & FA & DoA & DR & FR & DoR IVi \\
\hline 1 & $\begin{array}{l}\text { Pantanula } \\
\text { americana }\end{array}$ & 67 & 55,83 & 100,0 & 8,2627 & 11,40 & 3,74 & 25,54 & 40,68 \\
\hline 2 & $\begin{array}{l}\text { Diatenopteryx } \\
\text { sorbifolia }\end{array}$ & 33 & 27,50 & 100,0 & 2,4327 & 5,62 & 3,74 & 7,52 & 16,88 \\
\hline 3 & $\begin{array}{c}\text { Helietta } \\
\text { logifoliada }\end{array}$ & 38 & 31,67 & 87,5 & 1,4328 & 6,47 & 3,27 & 4,43 & 14,17 \\
\hline 4 & $\begin{array}{c}\text { Luehea } \\
\text { divaricata }\end{array}$ & 21 & 17,50 & 75,0 & 2,1237 & 3,57 & 2,80 & 6,56 & 12,93 \\
\hline 5 & $\begin{array}{l}\text { Holocalyx } \\
\text { balansae }\end{array}$ & 15 & 12,50 & 5,0 & 1,5894 & 2,55 & 2,80 & 4,91 & 10,26 \\
\hline 6 & $\begin{array}{l}\text { Chryssophyllum } \\
\text { gonocarpum }\end{array}$ & 27 & 22,50 & 87,5 & 0,5963 & 4,60 & 3,27 & 1,84 & 9,71 \\
\hline 7 & Indeterminadas & 21 & 17,50 & 87,5 & 0,3357 & 3,57 & 3,27 & 1,04 & 7,88 \\
\hline 8 & $\begin{array}{c}\text { Nectandra } \\
\text { megapotanica }\end{array}$ & 18 & 15,0 & 62,5 & 0,7111 & 3,06 & 2,34 & 2,20 & 7,60 \\
\hline 9 & $\begin{array}{c}\text { Lonchocarpus } \\
\text { sp. }\end{array}$ & 17 & 14,17 & 87,5 & 0,3719 & 2,89 & 3,27 & 1,15 & 7,31 \\
\hline 10 & $\begin{array}{l}\text { Machaerium } \\
\text { stipitatum }\end{array}$ & 13 & 10,3 & 75,0 & 0,645 & 2,21 & 2,80 & 2,12 & 7,13 \\
\hline 11 & $\begin{array}{c}\text { Syagrus } \\
\text { romanzoffiana }\end{array}$ & 13 & 10,83 & 75,0 & 0,5028 & 2,21 & 12,80 & 1,55 & 6,56 \\
\hline 12 & $\begin{array}{l}\text { Ruprechtia } \\
\text { laxiflora }\end{array}$ & 6 & 5,00 & 25,0 & 1,4695 & 1,02 & 0,93 & 4,54 & 6,49 \\
\hline
\end{tabular}

Ci. Fl., v. 5, n. 1, 1995 
TABELA 1: Continuação...

TABLE 1: Continued...

\begin{tabular}{|c|c|c|c|c|c|c|c|c|c|c|}
\hline Pos & Espécie & \multicolumn{2}{|c|}{ N. ind } & DA & FA & DoA & DR & FR & DoR & IVi \\
\hline 13 & $\begin{array}{l}\text { Nectrandra } \\
\text { lanceolata }\end{array}$ & 12 & 10,00 & 62,5 & 0,5524 & 2,04 & 2,34 & 1,71 & & 6,09 \\
\hline 14 & $\begin{array}{c}\text { Campomanesia } \\
\text { xanthocarpa }\end{array}$ & 11 & 9,17 & 75,0 & 0,3514 & 1,87 & 2,80 & 1,09 & & 5,76 \\
\hline 15 & $\begin{array}{c}\text { Myrcianthes } \\
\text { pungens }\end{array}$ & 10 & 8,33 & 50,0 & 0,5999 & 1,70 & 1,87 & 1,85 & & 5,42 \\
\hline 16 & $\begin{array}{l}\text { Chrysophyllum } \\
\text { marginatum }\end{array}$ & 13 & 10,83 & 62,5 & 0,2348 & 2,21 & 2,34 & 0,73 & & 5,28 \\
\hline 17 & $\begin{array}{l}\text { Myrciaria } \\
\text { rivularis }\end{array}$ & 12 & 10,00 & 62,5 & 0,2725 & 2,04 & 2,34 & 0,84 & & 5,22 \\
\hline 18 & $\begin{array}{c}\text { Pilocarpus } \\
\text { pennatifolius }\end{array}$ & 15 & 12,50 & 50,0 & 0,2450 & 2,55 & 1,87 & 0,76 & & 5,18 \\
\hline 19 & $\begin{array}{l}\text { Sebastiania } \\
\text { brasiliensis }\end{array}$ & 13 & 10,83 & 50,0 & 0,2619 & 2,21 & 1,87 & 0,81 & & 4,89 \\
\hline 20 & Cedrela fissilis & 10 & 8,33 & 37,5 & 0,5660 & 1,70 & 1,40 & 1,75 & & 4,85 \\
\hline 21 & $\begin{array}{l}\text { Cuponia } \\
\text { vernalis }\end{array}$ & 12 & 10,00 & 37,5 & 0,4402 & 2,04 & 1,40 & 1,36 & & 4,80 \\
\hline 22 & $\begin{array}{l}\text { Matayba } \\
\text { elaeagnoides }\end{array}$ & 12 & 10,00 & 50,0 & 0,2861 & 2,04 & 1,87 & 0,88 & & 4,79 \\
\hline 23 & $\begin{array}{l}\text { Myrocarpus } \\
\text { frondosus }\end{array}$ & 8 & 6,67 & 62,5 & 0,3199 & 1,36 & 2,34 & 0,99 & & 4,69 \\
\hline 24 & $\begin{array}{l}\text { Parpiptadenia } \\
\text { rígida }\end{array}$ & 7 & 5,83 & 37,5 & 0,6657 & 1,19 & 1,40 & 2,06 & & 4,65 \\
\hline 25 & Allophylus edulis & 10 & 8,33 & 50,0 & 0,3208 & 1,70 & 1,87 & 0,99 & & 4,56 \\
\hline 26 & $\begin{array}{l}\text { Cabralea } \\
\text { canjerana }\end{array}$ & 11 & 9,17 & 37,5 & 0,4051 & 1,87 & 1,40 & 1,25 & & 4,52 \\
\hline 27 & $\begin{array}{c}\text { Ficus } \\
\text { luschnathiana }\end{array}$ & & 6,67 & 37,5 & 0,5061 & 1,36 & 1,40 & 1,57 & & 4,33 \\
\hline 28 & Trichilia catigua & 10 & 8,33 & 50,0 & 0,1414 & 1,70 & 1,87 & 0,44 & & 4,01 \\
\hline 29 & $\begin{array}{c}\text { Tabebuia } \\
\text { impetiginosa }\end{array}$ & 6 & 5,00 & 37,5 & 0,5108 & 1,02 & 1,40 & 1,58 & & 4,00 \\
\hline 30 & Ocatea $s p$ & 6 & 5,00 & 37,5 & 0,4478 & 1,02 & 1,40 & 1,38 & & 3,80 \\
\hline 31 & Trichilia elegans & 6 & 5,00 & 62,5 & 0,0712 & 1,02 & 2,34 & 0,22 & & 3,58 \\
\hline
\end{tabular}

Continua... 
TABELA 2: Continuação...

TABLE 2: Continued...

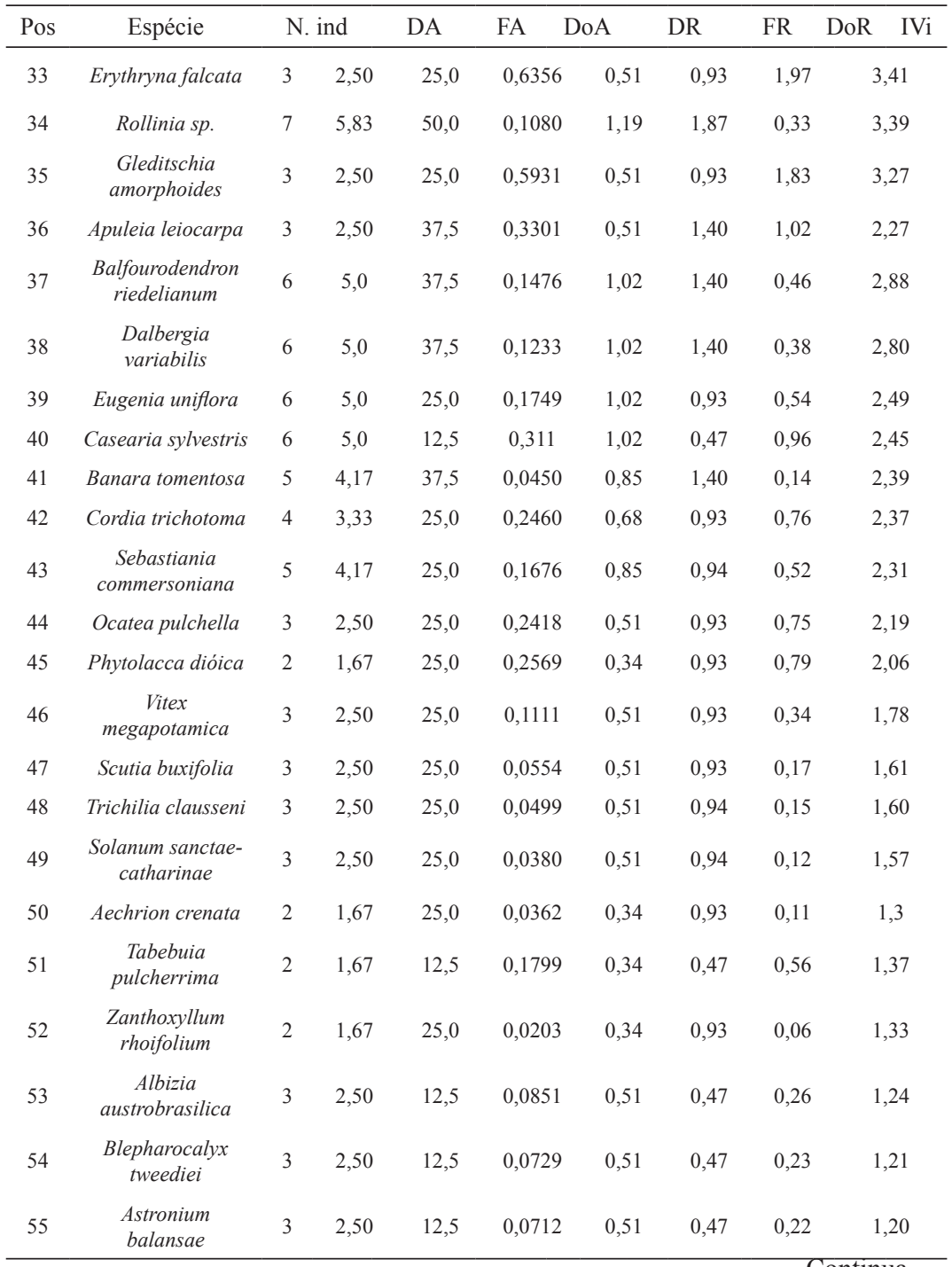


TABELA 2: Continuação...

TABLE 2: Continued...

\begin{tabular}{|c|c|c|c|c|c|c|c|c|c|}
\hline Pos & Espécie & & & $\mathrm{DA}$ & FA & DoA & DR & FR & DoR \\
\hline 57 & Tabebuia alba & 2 & 1,67 & 12,5 & 0,0811 & 0,34 & 0,47 & 0,09 & 0,90 \\
\hline 58 & $\begin{array}{c}\text { Guettarda } \\
\text { uruguensis }\end{array}$ & 2 & 1,67 & 12,5 & 0,0299 & 0,34 & 0,47 & 0,09 & 0,90 \\
\hline 59 & Cordia ecalyculata & 1 & 0,83 & 12,5 & 0,0316 & 0,17 & 0,47 & 0,10 & 0,74 \\
\hline 60 & Styrax leprosus & 1 & 0,83 & 12,5 & 0,0325 & 0,17 & 0,47 & 0,10 & 0,74 \\
\hline 61 & $\begin{array}{c}\text { Britoa } \\
\text { guazumefolia }\end{array}$ & 1 & 0,83 & 12,5 & 0,0172 & 0,17 & 0,47 & 0,05 & 0,69 \\
\hline 62 & Rapanea umbellata & 1 & 0,83 & 12,5 & 0,0153 & 0,17 & 0,47 & 0,05 & 0,69 \\
\hline 63 & $\begin{array}{l}\text { Eugenia } \\
\text { involucrata }\end{array}$ & 1 & 0,83 & 12,5 & 0,0106 & 0,17 & 0,47 & 0,03 & 0,67 \\
\hline 64 & Inga uruguensis & 1 & 0,83 & 12,5 & 0,0103 & 0,17 & 0,47 & 0,03 & 0,67 \\
\hline 65 & $\begin{array}{c}\text { Sorocea } \\
\text { bonplandii }\end{array}$ & 1 & 0,83 & 12,5 & 0,0101 & 0,17 & 0,47 & 0,03 & 0,67 \\
\hline 66 & Zanthoxyllum sp. & 1 & 0,83 & 12,5 & 0,0101 & 0,17 & 0,47 & 0,03 & 0,67 \\
\hline 67 & Rapanea sp. & 1 & 0,83 & 12,5 & 0,006 & 0,17 & 0,47 & 0,02 & 0,66 \\
\hline
\end{tabular}

Em que: N. ind- número de indivíduos amostrados; DA-densidade absoluta (arv/ ha); FA- freqüência absoluta(\%); DoA- dominância absoluta( $\left.\mathrm{m}^{2} / \mathrm{ha}\right)$; DR- densidade relativa(\%);FR-frequencia relativa(\%); DoR- dominância relativa(\%); IVI- índices de valor de importância ;

Os indivíduos indeterminados pertencem a diversas espécies, que não puderam ser identificados. Desta maneira foram agrupados na Tabela 2 como uma classe única. 


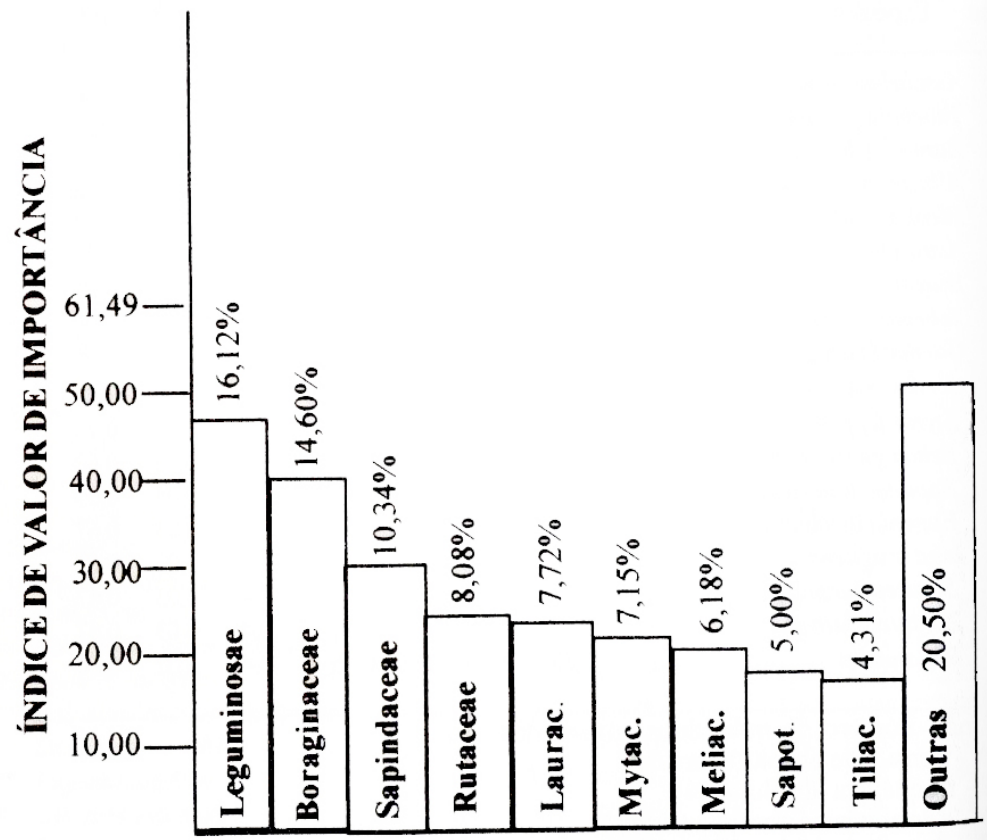

FAMÍLIAS

FIGURA 4: Relação das famílias que apresentam os maiores IVI.

FIGURE 4: List of the families with the highest IVI values. 


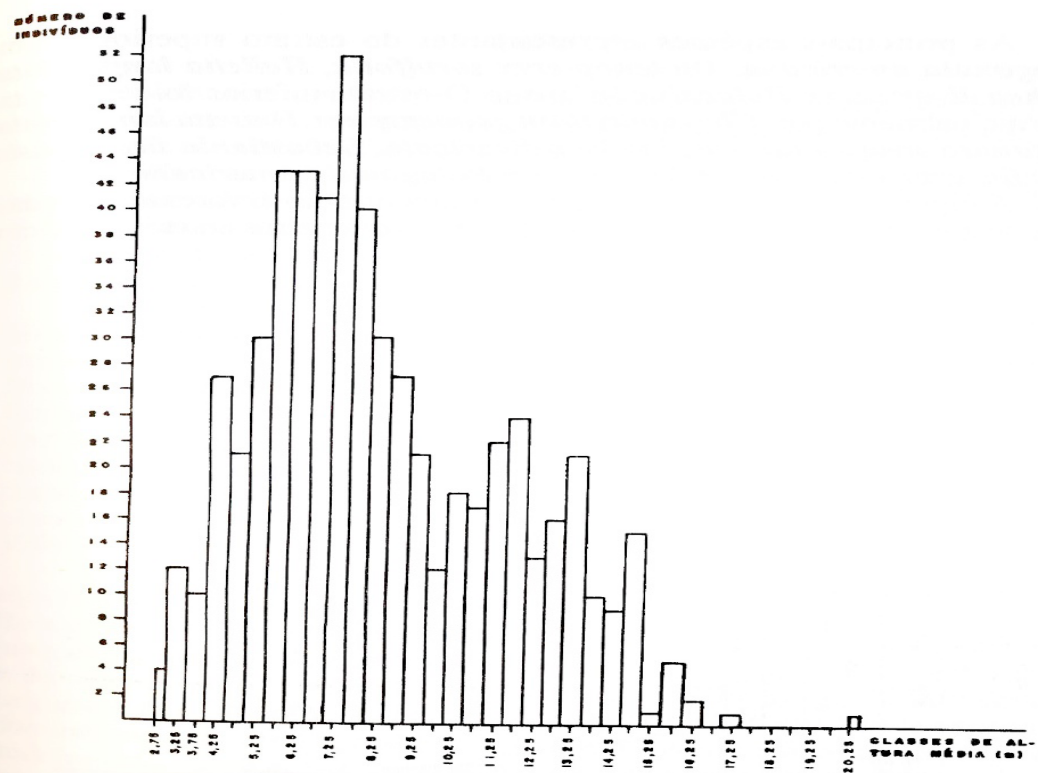

FIGURA 5: Distribuição de frequência das classes de altura média das árvores amostradas a partir de $30 \mathrm{~cm}$ de CAP. Amostra de 1,2 ha.

FIGURE 5: Frequency distribution of the classes of average height of the sampled trees from $30 \mathrm{~cm}$ of CAP. Sample of $1.2 \mathrm{ha}$.

As principais espécies representantes do estrato superior foram Patagonula americana, Diatenopteryx sorbifolia, Helietta longifoliata, Luehea divaricata e Holocalyx balansae. O estrato inferior foi representado principalmente por: Chrysophyllum gonocarpum, Helietta longifoliata, Nectandra megapotamica, Luehea divaricata, Sebastiania brasiliensis, Lonchocarpus sp., Allophylus edulis e Patagonula americana.

A Figura 6 mostra a distribuição de diâmetros das arvores amostradas. Nota-se que $63,3 \%$ dos indivíduos apresentaram diâmetro entre 9,5 e $25 \mathrm{~cm}$. 


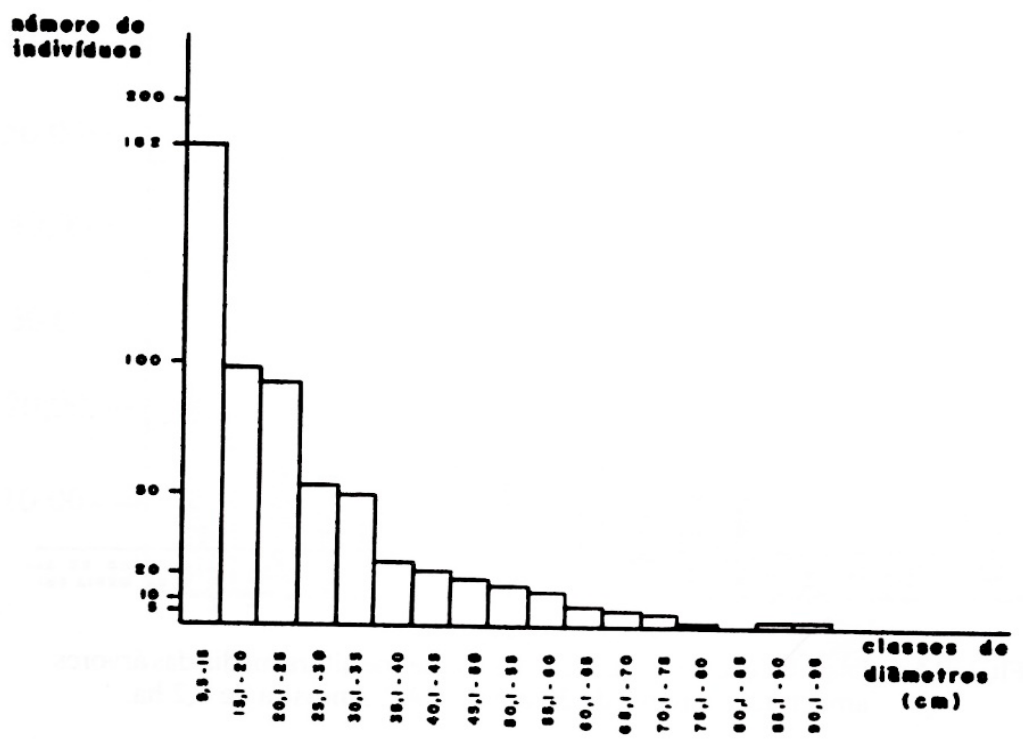

FIGURA 6: Distribuição de frequência das classes de diametro dos indivíduos amostrados a partir de $30 \mathrm{~cm}$ de CAP. Amostra de 1,2ha.

FIGURE 6: Frequency distribution of the classes of diameter of the sampled individuals from $30 \mathrm{~cm}$ of CAP. Sample of 1.2ha.

\section{DISCUSSÃO}

Levantamentos florísticos e fitossociológicos realizados em floresta estacional decidual ocorrente na Fralda da Serra Geral (DURLO et al., 1982; LONGUI et al., 1982, 1986; SILVA \& LONGHI, 1989; TABARELLI, 1992; VACCARO et al., 1992) demonstraram ser a família Leguminosae a mais rica das espécies arbóreas, figurando a seguir Myrtaceae, Lauraceae, Meliaceae e Euphorbiaceae. No Parque Estadual do Turvo, Tenente Portela, DIAS et al. (1992) encontraram como famílias mais ricas em espécies no componente arbóreo Leguminosae, Lauraceae, Euphorbiaceae e Meliaceae.

No presente estudo, embora não tenha sido possível classificar taxonomicamente $3,6 \%$ dos indivíduos amostradoa, as famílias mais ricas em espécies foram Leguminosae (11 espécies), Myrtaceae (7) e Lauraceae, 
Meliaceae e Rutaceae (4 espécies cada). Desta forma, em termos de riqueza florística por família, a composição de algumas áreas remenescentes da floresta do Alto Uruguai, entre os rios Ijui e Turvo, é semelhante a das demais florestas estacionais do estado do Rio Grande do Sul. Nestas, destaca-se Legumonosae como a família mais rica em espécies no componente arbóreo, fato este também observado para as florestas do interior do estado de São Paulo (VIEIRA et al., 1989).

Quanto ao número de indivíduos por família (Figura 3), não há diferença significativa entre as mais representadas, que são: Leguminosae (79 indivíduos), Boraginaceae (72), Sapindaceae (67) e Rutaceae (62). Porém, a analise conjunta da distribuição do numero de indivíduos (Figura 3), do numero de espécies e da importância (Figura 4) por família, indicam existir dominância da Leguminosae, que obteve o primeiro lugar em cada um destes aspectos. A dominância desta família poderia ser explicada admitindo-se que a presença de nódulos radiculares na maioria de suas espécies atuaria como um mecanismo de retenção e transferência de nitrogênio no ecossistema (MARTINS, 1979).

CUNHA et al. (1993) encontraram uma alta concentração de nitrogênio numa floresta estacional decidual em Santa Maria (RS), o que atribuíram a abundancia de espécies da família Leguminosae.

$\mathrm{Na}$ Tabela 2, Patagonula americana se apresenta em destaque na posição de IVI, em função dos altos valores de densidade, frequência e dominância de sua população. Esta espécie, embora apresente uma grande plasticidade de comportamento, ocorrendo em vários estádios de sucessão florestal, é considerada uma espécie pioneira heliófila, com elevada agressividade sub a subsere (SMITH, 1970; KLEIN, 1984). Ainda que vários fatores bióticos e abióticos possam colaborar para os altos valores de abundancia obtidos por esta espécie, acredita-se que a abertura de clareiras através de abates seletivos realizados no passado tenham propiciado tal fato.

Ao analisar a Figura 4, nota-se que a ordenação das famílias segue a da Figura 2, ou seja, há uma relação estreita entre o numero de indivíduos amostrados por determinada família e a importância desta na estrutura da floresta.

Observando a Figura 5, percebe-se que a frequência de altura média é maior em torno de 7,5 m. São raras as árvores com altura média superior a $15 \mathrm{~m}$, e apenas um indivíduo ocorre como emergente. A comparação da estrutura vertical obtida com a estratificação citada por KLEIN (1972), leva 
a supor que houve uma retirada das árvores altas e emergentes da floresta.

A Figura 6 revela uma alta frequência de arvores com diâmetros pequenos. Nas classes superiores, entre 75 e $95 \mathrm{~cm}$ ocorrem apenas cinco indivíduos, sendo três de Patagonula americana $(76,4 \mathrm{~cm} ; 87,5 \mathrm{~cm}$ e 92,3 $\mathrm{cm})$, um Erythryna falcata $(85,9 \mathrm{~cm})$ e um Ruprechtia laxiflora $(93,6 \mathrm{~cm})$.

Pelo exposto, percebe-se que os fragmentos florestais analisados estão se regenerando de perturbações sofridas no passado. A ausência de indivíduos de grande porte pertencentes a espécies produtoras de madeiras nobres reforçam esta colocação.

\section{REFERÊNCIAS BIBLIOGRÁFICAS}

ALBUQUERQUE, E.P. de. Sobre o desaparecimento da fauna da região do Alto Uruguai e a importância do Parque Florestal Estadual do Turvo na sua preservação. Roessléria, Porto Alegre, v.1, p.143-149, 1977.

ALBUQUERQUE, E.P. de. Considerações sobre a necessidade de pesquisa em conservação biológica nos parques estaduais do Rio Grande do Sul. Roessléria, Porto Alegre, v.7, p.200-205, 1985.

BLACK, P. et al. Levantamento florístico do Parque Estadual do Turvo, Tenente Portela, Rio Grande do Sul, Brasil. Roessléria, Porto Alegre, v.7, p.69-94, 1985.

BRASIL. Ministério da Agricultura. Instituto Brasileiro de Desenvolvimento Florestal. Inventário florestal nacional: floresta nativa, Rio Grande do Sul. Brasília: 1983.

BRASIL. Ministério da Agricultura. Divisão Pedológica. Levantamento de reconhecimento dos solos do estado do Rio Grande do Sul. Recife: 1973. (Boletim técnico, 30).

CUNHA, G.C. da. et al. Dinamica nutricional em floresta estacional decidual, com ênfase aos minerais provenientes da deposição da serapilheira. Ciência florestal, Santa Maria, v.3, n.1, p.35-64, 1993.

DIAS, L.L. da, et al. Levantamento florístico de área de mata subtropical no Parque Estadual do Turvo, Tenente Portela, RS. In: Rev. Inst. Flor., São Paulo, v.4, pt.2, p.339-346, 1992. (Edição Especial).

DURLO, M.A.; MARCHIORI, J.N.C.; LONGHI, S.J. A composição e estrutura da mata secundária no vale do rio Jacuí, RS. Ciência e Natura, Santa Maria, v.4, p.129-139, 1982.

FORTES, A.B. Aspectos fisiográficos, demográficos e econômicos do Rio 
Grande do Sul. Porto Alegre: Globo, 1956.

IBGE. Diretoria de Geociências. Mapa de vegetação do Brasil. 2. ed. Rio de Janeiro, 1993. 1 mapa de $101 \times 108 \mathrm{~cm}$. Escala 1: 5.000.000.

IRGANG, B.E. A mata do Alto Uruguai no Rio Grande do Sul. Ciência e Cultura, Porto Alegre, v.32, n.3, p.323-324, 1980.

KLEIN, R.M. Árvores nativas da floresta subtropical do Alto Uruguai. Sellowia, Itajaí, v.24, p.9-62, 1972.

KLEIN, R.M. Síntese ecológica da floresta estacional da bacia do rio Jacuí e importancia do reflorestamento com essências nativas (RS). In: CONGRESSO FLORESTAL ESTADUAL, 5. 1984. Nova Prata. Anais... Nova Prata, 1984. p.265-278.

LEITE, P.F.; KLEIN, R.M. Vegetação. In: MESQUITA, O.V. (Coord.). Geografia do Brasil: Região Sul. Rio de Janeiro: IBGE. 1990. v.2, p.113-150. LONGHI, S.J.; DURLO, M.A.; MARCHIORI, J.N.C. A vegetação da mata ribeirinha no curso médio do rio Jacuí, RS. Ciência e Natura, Santa Maria, v.4, p.151-161, 1882.

LONGHI, S.J.; SCHORN, L.A. Diferenciação dos tipos florestais do morro Botucaraí, em Candelária, Rio Grande do Sul. Acta For. Bras., Curitiba, v.1, p.99-114, 1986.

MAECHIORI, J.N.C. Florestas nativas privadas: uma analise fito geográfica histórica do problema do Rio Grande do Sul. In: SEMINARIO SOBRE A SITUAÇÃO FLORESTAL DO RIO GRANDE DO SUL, 1. 1991, Santa Maria. Anais... Santa Maria, 1991. p.21-27.

MARTINS, F.R. O método de quadrantes e a fitossociologia de uma floresta residual do interior do estado de São Paulo. São Paulo: USP. 1979. 239p. Tese (Doutorado) - Instituto de Biociências, Universidade de São Paulo. MUELLER-DOMBOIS, D.; ELLENBERG, H. Aims and methods of vegetation ecology. New York: Willey and Sons, 1974.

PASTORE, U.; RANGELFILHO,A.L.R. Vegetação; as regiões fitoecológicas, sua natureza e seus recursos econômicos. Estudo fitogeográfico. Folha Uruguaiana. In: FOLHA SH-22 Porto Alegre e parte das folhas SH-21 Uruguaiana e SI-22 lagoa Mirim. Rio de Janeiro: IBGE, 1986. p.541-632. (Levantamento de recursos Naturais, 33).

RAMBO, B. Der Regenwald am oberen Uruguay. Sellowia, Itajaí, v.7, p.183233, 1956a.

RAMBO, B. A fisionomia do Rio Grande do Sul. Porto Alegre: Selbach, 1956b. RIO GRANDE DO SUL. Secretaria da Agricultura. Plano de manejo 
do Parque Estadual do turvo. Porto Alegre: 1980.

SILVA, L.B. da.; LONGHI, S.J. Aspectos florísticos e fitossociológicos do morro Link, santa Maria, RS. In: SEMANA DO MEIO AMBIENTE, 3., 1989, Piracicaba. Anais... Piracicaba, 1989. p.177-207.

SMITH, L.B. Boragináceas. In: Flora Ilustrada catarinense. Itajaí: Herbário barbosa Rodrigues, 1970.

TABARELLI, M. Flora arbórea da floresta estacional baixo-montana no município de Santa Maria, RS, Brasil. Rev. Inst. Flor., São Paulo, v.4, pt.1, p.260-268, 1992; (Edição Especial).

VACCARO, S.; HESS, A.F.; LONGHI, S.J. Estudo da composição florística e estrutura da vegetação arbórea da sede do município de Santa Tereza - RS. In: CONGRESSO FLORESTAL ESTADUAL, 7., 1992. Nova Prata. Anais... Nova Prata, 1992. p.331-353.

VASCONCELLOS, J.M. de O.; DIAS, L.L.; SILVA, C.P. da.; SOBRAL, M. Fitossociologia de uma área de mata subtropical no Parque estadual do Turvo - RS. Rev. Inst. Flor., São Paulo, v.4, pt.1, p.252-259, 1992. (Edição especial).

VIEIRA, M.G.L. et al. Composição florística e estrutura fitossociológica da vegetação arbórea do Parque Estadual de Vaçununga, Santa Rita do Passa Quatro (SP). II-Gleba Capetinga Oeste. Rev. Inst. Flor., São Paulo, v.1, n.1, p.135-159, 1989.

WALTER, $\mathrm{H}$. Climatic diagrams as a means to comprehend the various climatic types for ecological and agricultural purposes. In: RUTTER, A.J., WHITEHEAD, F.H. The water relations of plants. London: Blackwell Scientific, 1963. p.3-9. 